\title{
B2DI \\ A Bayesian BDI Agent Model with Causal Belief Updating based on MSBN
}

\author{
Álvaro Carrera and Carlos A. Iglesias \\ Departamento de Ingeniería de Sistemas Telemáticos, Universidad Politécnica de Madrid, Madrid, Spain
}

Keywords: Distributed, Agents, Bayesian, BDI, MSBN, Belief, Sharing.

\begin{abstract}
In this paper, we introduce B2DI model that extends BDI model to perform Bayesian inference under uncertainty. For scalability and flexibility purposes, Multiply Sectioned Bayesian Network (MSBN) technology has been selected and adapted to BDI agent reasoning. A belief update mechanism has been defined for agents, whose belief models are connected by public shared beliefs, and the certainty of these beliefs is updated based on MSBN. The classical BDI agent architecture has been extended in order to manage uncertainty using Bayesian reasoning. The resulting extended model, so-called B2DI, proposes a new control loop. The proposed B2DI model has been evaluated in a network fault diagnosis scenario. The evaluation has compared this model with two previously developed agent models. The evaluation has been carried out with a real testbed diagnosis scenario using JADEX. As a result, the proposed model exhibits significant improvements in the cost and time required to carry out a reliable diagnosis.
\end{abstract}

\section{INTRODUCTION}

Many problems of our everyday life are connected in huge information networks both computer and human networks. Some of these problems are too complex to be completely modelled and described. Thus, these problems have been solved through distributed systems. Multi-agent systems (MAS) provide a suitable abstraction level for dealing with the complexity of large scale, open, heterogeneous, and evolving scenarios.

In recent past, several works have studied different approaches (Fagundes et al., 2009; Casali et al., 2011) to reason with uncertainty in a single agent. In this work, an approach to deal with uncertainty in a distributed way to achieve collaborative goal deliberation based on a BDI MAS is presented. Multiply Sectioned Bayesian Network (Xiang et al., 1993) (MSBN) approach is proposed to handle uncertainty in a distributed way, to keep coherence in the inference process and to allow flexibility in the multi-agent system. We adopt the terminology by Xiang (Xiang et al., 1993; Xiang, 2002; Xiang et al., 2006) in his work.

The reminder of this paper is structured as follows. Sect. 2 presents the proposed Bayesian agent model to perform distributed causal inference of beliefs. Sect. 3 presents the evaluation of the model on the basis of a real testbed diagnosis scenario. Finally, Sect. 4 presents several important related works and Sect. 5 presents the reached conclusions and possible future research areas.

\section{B2DI: BAYESIAN BDI AGENT MODEL}

This section presents the extended BDI model to perform distributed causal inference using MSBNs.

Definition 1. Causal Model $C M$ is a Bayesian Network used by an agent to perform distributed causal inference. $C M$ is a subnet that is part of a MSBN. The agent uses this $C M$ to perform belief updating depending on the current available data.

The proposed model is based on sharing a distributed causal model, that is partitioned among the agents. In this partitioning, beliefs can be private or shared with other agents.

Fig. 1 shows the block diagram of a B2DI Agent. A B2DI agent works with a modified BDI architecture. This model updates beliefs based on a distributed causal inference through MSBN.

A B2DI agent starts its behaviour with initial beliefs and intentions (Alg. 1). These beliefs are added to the $C M$ and synchronized (Algs. 2 and 3) with other 


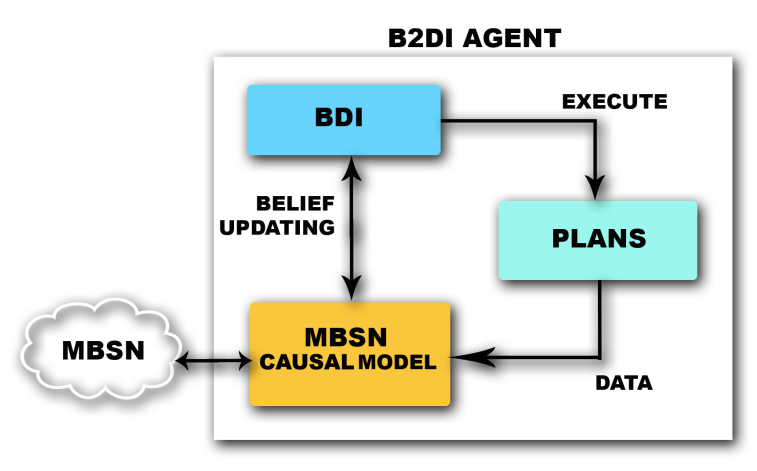

Figure 1: B2DI Agent Model.

agents through MSBN. After deliberation process, a plan is selected and executed. Any data obtained after the execution of a plan is added to the $C M$.

Alg. 1 shows the agent control loop proposed in this work. Several functions are used in this algorithm. This control loop is similar to the previous classic BDI model, but it has been extended with the following functions: connect function (Alg. 2), belief initialization function (Alg. 3), update notification function (Alg. 4), belief update function (Alg. 5) and communicate belief function (Alg. 6).

One new variable is added in this proposed control loop algorithm (Alg. 1). Mod CM represents the Causal Model (Def. 1) of the agent.

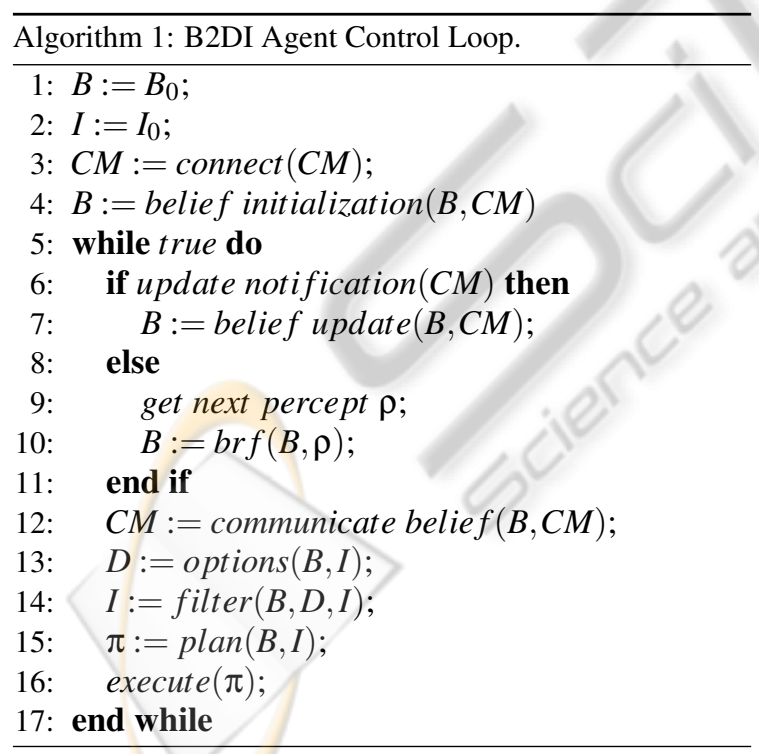

Alg. 2 presents connect function to set-up the agent $C M$ (a subnet) with the distributed inference engine. This function consists of two steps: moralization and triangulation to achieve coherence.

Once the subnet is ready, the belief initialization function is called to synchronize the shared knowledge. First of all, the agent receives a notification of

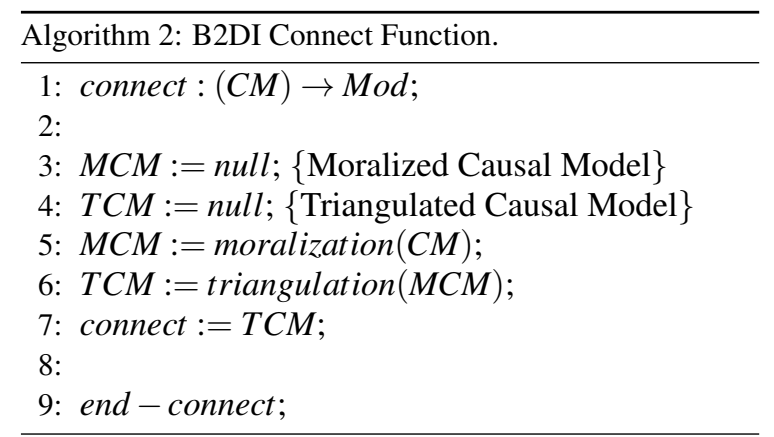

belief updating and it updates its own beliefs through local inference process. After this, the agent propagates its beliefs to other agents.

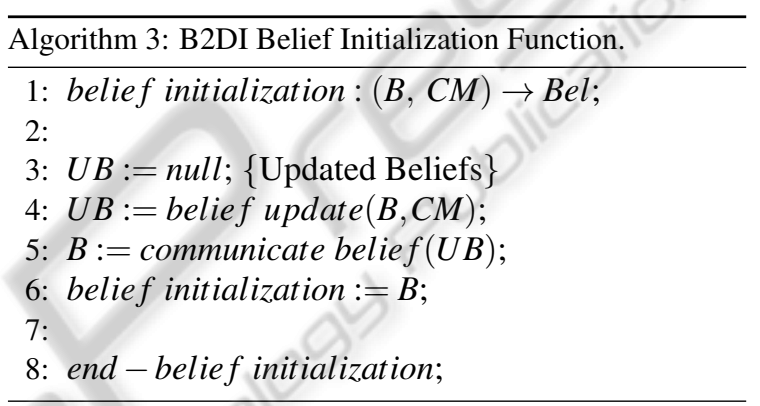

Alg. 4 shows the update notification function. This function simply notifies when it exists a change in the set of shared beliefs.

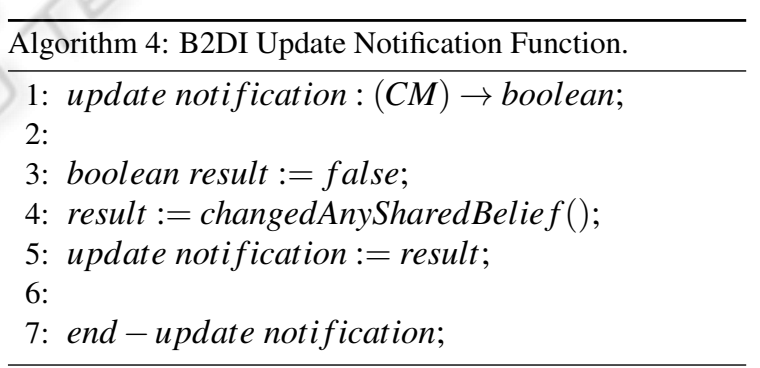

Alg. 5 shows the process required to receive believes. First of all, new beliefs are received through linkages. Then, this beliefs are locally propagated in the subnet $C M$ (Def. 1) of the agent.

When local beliefs are modified or external beliefs are received, the agent must communicate this updates to the rest of the MSBN. This process is composed of two steps: collect beliefs and distribute beliefs.

\section{EVALUATION}

The evaluation has been carried out based on a bench- 
mark for a real diagnosis scenario of the $R \& D$ project "Magneto" (Arozarena et al., 2010). This scenario consists of a P2P streaming scenario where a user provides multimedia content and another user consumes this content. Many faults may occur both in connection and in services. The system is designed to provide, to an end-user or an operator, the result of the diagnosis made upon receipt of a notification of a symptom of failure. We have clustered all possible diagnoses of this scenario in 13 diagnosis cases to simplify comparison and shown results.

The evaluation consists of the comparison of three models that use Bayesian networks to perform causal inference to diagnose faults:
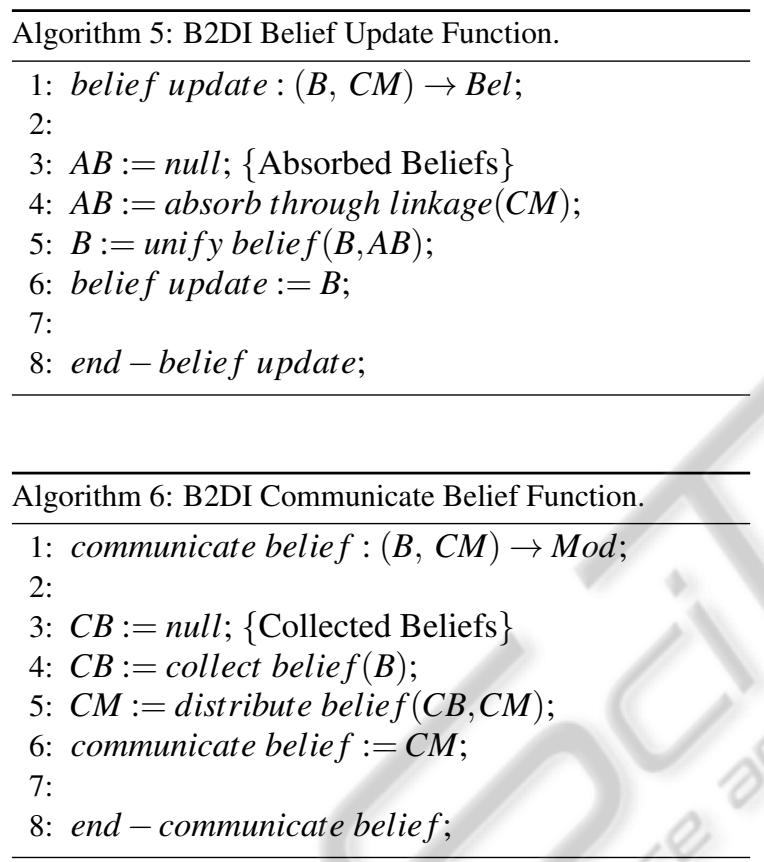

- Model 1 (Belief Flooding): All agents have Bayesian Networks with duplicated part of their causal model $C M$ to keep coherence in the process inference. Agents share beliefs about the available information of the current environment. When new information is received from other agents, it is propagated to the rest of the agents to assure the coherence of the distributed reasoning process. After this propagation, each agent continues with its own behaviour and shares its beliefs if it is required.

- Model 2 (Filtered Belief Flooding): Same than Model 1 but this time when new information is received from other agents, it is processed and filtered. Then, the agent takes its own decision and execute a selected plan. Finally, only filtered information is propagated to its neighbours.
- Model 3 (B2DI, Belief Sharing based on Causal Dependencies): This model represents the proposed B2DI model. These agents share their beliefs only with other agents which are interested in them. When new information is available, it is automatically propagated through MSBN mechanism.

Table 1 shows the evaluation results in several columns. MTTD (FitzGerald and Dennis, 2008) (Mean Time to Diagnose) usually is the average number of minutes until the root cause of the failure is correctly diagnosed; but, in this table, we show this time rounded in seconds.

Model 3 presents a reduction of $48 \%$ in time and $37 \%$ in number of executed tests comparing with Model 1 and a reduction of $40 \%$ in time and $60 \%$ in number of executed tests comparing with Model 2.

\section{RELATED WORK}

Although uncertain belief representation in BDI-like systems have been studied in AI over recent past (Fagundes et al., 2009; Casali et al., 2011), this work is novel in several respects. It proposes transparently sharing beliefs methods through MSBNs (Xiang, 2002), i.e. an agent do not have to send its beliefs through a message. MSBN model assures consistency and coherence during the inference process. This MSBN model is embedded in the proposed B2DI model (Sect. 2).

Furthermore, MSBN offers dynamically reconfiguration (Xiang et al., 2006) if any agent is born or dies in the environment. Thus, all agents that share any set of beliefs can reconfigure its causal model to link to the global inference mechanism (MSBN) even if an intermediate agent disappears.

The use of distributed causal models to fault diagnosis has been previously studied (Bocaniala and da Costa, 2004) and the proposed methodology to partition the global causal model in causally independent (d-separated) regions. But, that methodology only exposes how to fragment the causal model in smaller pieces. It does not deal with how the inference should be performed in a distributed way. Our proposed approach (B2DI agent model) uses distributed causal models and presents a framework to perform distributed inference. 
Table 1: MTTD and number of test comparison.

\begin{tabular}{|c|c|c|c|c|c|c|}
\hline \multirow{2}{*}{ Diagnosis case } & \multicolumn{3}{|c|}{ MTTD (in seconds) } & \multicolumn{3}{c|}{ Number of executed tests } \\
\cline { 2 - 7 } & Model 1 & Model 2 & Model 3 & Model 1 & Model 2 & Model 3 \\
\hline Case 1 & 22 & 19 & 6 & 6 & 7 & 4 \\
\hline Case 2 & 9 & 8 & 4 & 5 & 2 & 2 \\
\hline Case 3 & 12 & 9 & 5 & 4 & 6 & 2 \\
\hline Case 4 & 15 & 10 & 9 & 6 & 9 & 4 \\
\hline Case 5 & 3 & 4 & 3 & 1 & 1 & 1 \\
\hline Case 6 & 16 & 15 & 5 & 4 & 8 & 2 \\
\hline Case 7 & 13 & 10 & 6 & 3 & 6 & 2 \\
\hline Case 8 & 15 & 14 & 7 & 3 & 9 & 2 \\
\hline Case 9 & 18 & 24 & 13 & 7 & 12 & 5 \\
\hline Case 10 & 15 & 19 & 13 & 5 & 8 & 3 \\
\hline Case 11 & 9 & 6 & 6 & 2 & 2 & 2 \\
\hline Case 12 & 13 & 9 & 7 & 3 & 5 & 2 \\
\hline Case 13 & 21 & 14 & 10 & 5 & 9 & 3 \\
\hline Average & 14.4 & 12.5 & 7.4 & 4.2 & 6.9 & 2.6 \\
\hline
\end{tabular}

\section{CONCLUSIONS AND FUTURE WORK}

This paper proposes a Bayesian BDI agent model with causal belief updating based on MSBN. This process implies a modification in the BDI control loop. In B2DI model, there are several additional operations to keep coherence and consistency in the sharing process. These additional operations in the control loop (see Alg. 1) allow to decrease the complexity of the belief sharing process. This sharing process is performed transparently through MSBN mechanisms and it has not to be implemented explicitly in the agent behaviour. MSBN mechanisms offer flexibility (reconfiguration capabilities) and robustness to allow new agents to join the belief sharing group and keep coherence in the reasoning process without any modification of the agent behaviour.

As future work, several paths can be explored. One interesting option is the extension of MSBN use for distributed goal deliberation. The sharing process offered by MSBN can be used to achieve common or private goals knowing intentions of other agents in a transparent way. Further, another option to work is a formal methodology to apply this model during the development of multi-agent systems. Based on graph theory, agents causal models (see Def. 1) could be dynamically generated from a global knowledge base, i.e. from a global causal model that deals with the domain knowledge. For future scenario, sensor networks will be applied. B2DI model meets the requirements of sensor networks, such as distributed data processing, robustness or reconfiguration capabilities during the inference process.

\section{REFERENCES}

Arozarena, P., Toribio, R., Kielthy, J., Quinn, K., and Zach, M. (2010). Probabilistic Fault Diagnosis in the MAGNETO Autonomic Control Loop. In 4th International Conference on Autonomous Infrastructure, Management and Security, pages 102-105. Springer.

Bocaniala, C. D. and da Costa, J. (2004). Novel framework for using causal models in distributed fault diagnosis. In Proceedings of Workshop on Advances in Control and Diagnosis, Karlsruhe, Germany, pages 142-147. Citeseer.

Casali, A., Godo, L., and Sierra, C. (2011). A graded BDI agent model to represent and reason about preferences. Artificial Intelligence, 175(7-8):1468-1478.

Fagundes, M., Vicari, R., and Coelho, H. (2009). Deliberation process in a BDI model with Bayesian networks. Agent Computing and Multi-Agent Systems, pages 207-218.

FitzGerald, J. and Dennis, A. (2008). Business Data Communications and Networking. John Wiley and Sons.

Xiang, Y. (2002). Probabilistic reasoning in multiagent systems: a graphical models approach. Cambridge University Press.

Xiang, Y., Jensen, F. V., and Chen, X. (2006). Inference in multiply sectioned Bayesian networks: methods and performance comparison. IEEE Transactions on Systems, Man and Cybernetics, Part B (Cybernetics), 36(3):546-558.

Xiang, Y., Poole, D., and Beddoes, M. P. (1993). Multiply sectioned bayesian networks and junction forests for large knowledge-based systems. Computational Intelligence, 9(2):171-220. 